\title{
GENERALIZED CONVEX FUNCTIONS AND SECOND ORDER DIFFERENTIAL INEQUALITIES
}

\author{
MAURICIO MATOS PEIXOTO
}

1. Introduction. A well known theorem states that a necessary and sufficient condition in order that the twice differentiable function $y(x), a<x<b$, be convex is that $y^{\prime \prime} \geqq 0$. The condition $y^{\prime \prime}>0$ is sufficient for the strict convexity of $y$.

In the present paper we show that if convexity is taken in the generalized sense of E. F. Beckenbach $[1,2],{ }^{1}$ a differential characterization of the above type can be obtained. As a particular case of a general theorem concerning second order differential inequalities we obtain a recent result of S. Tchaplygin, V. N. Petrov and J. E. Wilkins [3] concerning linear differential inequalities.

2. Generalized convexity. Let $\{F(x)\}$ be a family of real functions of the real variable $x$ defined for $a<x<b$ and such that:

(1) Each member of the family is a continuous function of $x$.

(2) Given in the $x y$-plane two arbitrary points $\left(x_{1}, y_{1}\right),\left(x_{2}, y_{2}\right)$ such that $a<x_{1}<x_{2}<b$, there is a unique member of the family passing through these two points, that is, such that its graph passes through these two points.

A function $\phi(x), a<x<b$, is said to be convex relative to the family $\{F(x)\}-a$ sub- $\{F(x)\}$ function in Beckenbach's notation-if, for arbitrary $x_{1}, x_{2}$ such that $a<x_{1}<x_{2}<b$, the member of the family, $F_{12}(x)$, which pasess through $\left[x_{1}, \phi\left(x_{1}\right)\right],\left[x_{2}, \phi\left(x_{2}\right)\right]$ is such that

$$
\phi(x) \leqq F_{12}(x) \text {, }
$$

$$
x_{1} \leqq x \leqq x_{2} \text {. }
$$

If we have

$$
\phi(x)<F_{12}(x), \quad x_{1}<x<x_{2},
$$

we say that $\phi(x)$ is strictly convex relative to the family $\{F(x)\}$ or else that it is a strictly sub- $\{F(x)\}$ function.

The ordinary convexity is obtained if we take as the family $\{F(x)\}$ the linear functions $m x+n$.

3. An auxiliary theorem. $\phi(x)$ being a sub- $\{F(x)\}$ function and $a<x_{0}<b$ we have proved elsewhere [4] the following theorem.

Theorem 1. There exist $D(x) \in\{F(x)\}, E(x) \in\{F(x)\}$ such that

Received by the editors April 9, 1948.

${ }^{1}$ Numbers in brackets refer to the bibliography at the end of the paper. 


$$
\begin{gathered}
D\left(x_{0}\right)=E\left(x_{0}\right)=\phi\left(x_{0}\right), \\
E(x) \leqq D(x) \leqq \phi(x), \\
D(x) \leqq E(x) \leqq \phi(x),
\end{gathered}
$$$$
x_{0}<x<b \text {, }
$$$$
a<x<x_{0} .
$$

(8) If $\phi^{\prime}\left(x_{0}\right), E^{\prime}\left(x_{0}\right), D^{\prime}\left(x_{0}\right)$ exist, then $E^{\prime}\left(x_{0}\right)=D^{\prime}\left(x_{0}\right)=\phi^{\prime}\left(x_{0}\right)$.

4. The family $\{F(x)\}$ as solutions of a differential equation. Consider on the strip $S, a<x<b,-\infty<y<+\infty$, the differential equation

$$
y^{\prime \prime}=G\left(x, y, y^{\prime}\right)
$$

and let us suppose:

(10) the function $G\left(x, y, y^{\prime}\right),(x, y) \in S,-\infty<y^{\prime}<+\infty$, is continuous;

(11) to each point $\left(x_{0}, y_{0}\right) \in S$ and $-\infty<y_{0}^{\prime}<+\infty$, there exists a unique solution $y(x)$ of (9) defined for $a<x<b$ such that

$$
y\left(x_{0}\right)=y_{0}, \quad y^{\prime}\left(x_{0}\right)=y_{0}^{\prime}
$$

and we shall assume the continuity of this solution with respect to the initial values $y_{0}, y_{0}^{\prime}$;

(12) given two distinct points belonging to $S$ there is a unique solution of (9) passing through these points.

For some special types of $G\left(x, y, y^{\prime}\right)$ it is possible to assure that this condition (12) holds [5, Chap. V, VI].

From now on $\{F(x)\}$ will designate the family formed by the solutions of (9). Now, let $\phi(x), a<x<b$, be a function with a continuous second derivative. Our main result is expressed by the following theorem.

Theorem 2. A necessary and sufficient condition that $\phi(x)$ be a sub$\{F(x)\}$ function is that

$$
\phi^{\prime \prime} \geqq G\left(x, \phi, \phi^{\prime}\right), \quad a<x<b .
$$

Proof. (a) The condition is necessary. Let $\varphi(x)$ be a sub- $\{F(x)\}$ function and consider a generic point $a<x_{0}<b$. Consider $\varphi(x)$ $\in\{F(x)\}$ such that

$$
\varphi\left(x_{0}\right)=\phi\left(x_{0}\right), \quad \varphi^{\prime}\left(x_{0}\right)=\phi^{\prime}\left(x_{0}\right) .
$$

By Theorem 1 and (11) we have

$$
\varphi(x)=D(x)=E(x) \leqq \phi(x), \quad a<x<b .
$$

We have by (14) 


$$
\begin{aligned}
\phi(x)-\varphi(x) & =\phi\left[x_{0}+\left(x-x_{0}\right)\right]-\varphi\left[x_{0}+\left(x-x_{0}\right)\right] \\
& =\frac{\left(x-x_{0}\right)^{2}}{2}\left[\phi^{\prime \prime}\left(x_{0}\right)-\varphi^{\prime \prime}\left(x_{0}\right)+\epsilon\right]
\end{aligned}
$$

where $\epsilon$ is infinitesimal with $\left|x-x_{0}\right|$.

Suppose

$$
\phi^{\prime \prime}\left(x_{0}\right)<\varphi^{\prime \prime}\left(x_{0}\right) .
$$

In this case we have, in a suitable neighborhood of $x_{0}$,

$$
\phi(x)<\varphi(x)
$$

which contradicts (15). As (17) is false, we must have, in virtue of (9) and (14),

$$
\phi^{\prime \prime}\left(x_{0}\right) \geqq \varphi^{\prime \prime}\left(x_{0}\right)=G\left[x_{0}, \phi\left(x_{0}\right), \phi^{\prime}\left(x_{0}\right)\right]
$$

for every $a<x_{0}<b$. Then (13) holds.

(b) The condition is sufficient. Suppose that (13) holds. The demonstration follows easily from the following lemmas:

Lemma 1. If (13) holds, to every $a<x_{0}<b$ there exists $a \nu>0$ such that

$$
\phi\left(x^{\prime}\right)-\bar{\varphi}\left(x^{\prime}\right) \geqq 0, \quad\left|x^{\prime}-x_{0}\right| \leqq \nu,\left|\bar{x}-x_{0}\right| \leqq \nu,
$$

where $\bar{\varphi}(x) \in\{F(x)\}$ is defined by

$$
\bar{\varphi}(\bar{x})=\phi(\bar{x}), \quad \bar{\varphi}^{\prime}(\bar{x})=\phi^{\prime}(\bar{x}) .
$$

In fact, we have

$$
\begin{aligned}
\phi\left(x^{\prime}\right)-\bar{\varphi}\left(x^{\prime}\right) & =\phi\left[\bar{x}+\left(x^{\prime}-\bar{x}\right)\right]-\bar{\varphi}\left[\bar{x}+\left(x^{\prime}-\bar{x}\right)\right] \\
& =\frac{\left(x^{\prime}-\bar{x}\right)^{2}}{2}\left[\phi^{\prime \prime}\left(x^{*}\right)-\bar{\varphi}^{\prime \prime}\left(x^{*}\right)\right], \\
x^{*} & =\bar{x}+\theta\left(x^{\prime}-\bar{x}\right), \quad 0<\theta<1 .
\end{aligned}
$$

As $\phi^{\prime \prime}(x)$ is a continuous function, given arbitrarily $\epsilon>0$, there exists $h>0$ such that

$$
\phi^{\prime \prime}\left(x^{*}\right)>\phi^{\prime \prime}\left(x_{0}\right)-\epsilon / 2, \quad\left|x^{\prime}-x_{0}\right| \leqq h,\left|\bar{x}-x_{0}\right| \leqq h .
$$

By the supposed continuity of the solutions of (9) relative to the initial values, there is an $h_{1}>0$ such that

$$
\left|\bar{\varphi}^{\prime \prime}\left(x^{*}\right)-\varphi^{\prime \prime}\left(x^{*}\right)\right|<\epsilon / 4, \quad\left|x^{\prime}-x_{0}\right| \leqq h_{1},\left|\bar{x}-x_{0}\right| \leqq h_{1},
$$
$\varphi(x) \in\{F(x)\}$ satisfying (14). 
By the continuity of $\varphi^{\prime \prime}(x)$, there is an $h_{2}>0$ such that

$$
\left|\varphi^{\prime \prime}\left(x^{*}\right)-\varphi^{\prime \prime}\left(x_{0}\right)\right|<\epsilon / 4, \quad\left|x^{\prime}-x_{0}\right| \leqq h_{2},\left|\bar{x}-x_{0}\right| \leqq h_{2} .
$$

If we put $\nu=\min \left(h, h_{1}, h_{2}, 2^{1 / 2} / 2\right)$ we have from (24) and (25)

$$
\bar{\varphi}^{\prime \prime}\left(x^{*}\right)<\varphi^{\prime \prime}\left(x_{0}\right)+\epsilon / 2, \quad\left|x^{\prime}-x_{0}\right| \leqq \nu,\left|\bar{x}-x_{0}\right| \leqq \nu .
$$

From (22), (23), (26) and (19) we have

$$
\begin{aligned}
\phi\left(x^{\prime}\right)-\bar{\varphi}\left(x^{\prime}\right) & >\frac{\left(x^{\prime}-\bar{x}\right)^{2}}{2}\left[\phi^{\prime \prime}\left(x_{0}\right)-\varphi^{\prime \prime}\left(x_{0}\right)-\epsilon\right] \\
& \geqq-\frac{\left(x^{\prime}-\bar{x}\right)^{2}}{2} \epsilon \geqq-\epsilon, \\
& \left|x^{\prime}-x_{0}\right| \leqq \nu,\left|\bar{x}-x_{0}\right| \leqq \nu .
\end{aligned}
$$

As $\epsilon$ is an arbitrarily chosen positive number, (20) holds.

Definition 1. Let us say that a closed interval of $(a, b)$ has the property $(\mathrm{P})$ when the graph of $\phi(x)$ in this interval lies above or on any integral curve of (9), which at a point of the arc of $\phi(x)$ corresponding to the interval has the same slope as $\phi(x)$.

In terms of this definition the above lemma states that any point $a<x_{0}<b$ is the center of a closed interval where the property (P) holds.

LemmA 2. The property (P) holds on every closed interval $[\alpha, \beta]$, $a<\alpha \leqq x \leqq \beta<b$.

In fact, by the application of Lemma 1 and the familiar HeineBorel theorem, we have that there are a finite number of closed inintervals covering $[\alpha, \beta]$ for which the property $(\mathrm{P})$ holds. Observe that the closed interval intersection of two closed intervals for which the property $(\mathrm{P})$ holds is another closed interval for which this property holds.

Then Lemma 2 is proved if we show that the closed interval union of two contiguous closed intervals having the property $(\mathrm{P})$ is another closed interval for which this property holds. But this is easy to see. Indeed, let $\left[x_{1}, x_{2}\right],\left[x_{2}, x_{3}\right], x_{1}<x_{2}<x_{3}$, be the two closed intervals.

We shall show that the property $(\mathrm{P})$ holds on $\left[x_{1}, x_{3}\right]$. Let, for instance, $x_{1} \leqq x_{0} \leqq x_{2}$, and $\varphi(x) \in\{F(x)\}$ be defined by (14). We have, as $\left[x_{1}, x_{2}\right]$ has the property $(\mathrm{P})$,

$$
\varphi(x) \leqq \phi(x), \quad x_{1} \leqq x \leqq x_{2} .
$$

Suppose 


$$
\varphi\left(x_{2}\right)=\phi\left(x_{2}\right) .
$$

Let us show that we must have

$$
\varphi^{\prime}\left(x_{2}\right)=\phi^{\prime}\left(x_{2}\right) .
$$

Indeed, according to (28), we must have

$$
\varphi^{\prime}\left(x_{2}\right) \geqq \phi^{\prime}\left(x_{2}\right) \text {. }
$$

We shall show that the hypothesis

$$
\varphi^{\prime}\left(x_{2}\right)>\phi^{\prime}\left(x_{2}\right)
$$

is false. In fact, suppose that $\psi(x) \in\{F(x)\}$ is such that

$$
\psi\left(x_{2}\right)=\phi\left(x_{2}\right), \quad \psi^{\prime}\left(x_{2}\right)=\phi^{\prime}\left(x_{2}\right) .
$$

Then, by property $(\mathrm{P})$, we have

$$
\psi(x) \leqq \phi(x),
$$$$
x_{1} \leqq x \leqq x_{3} .
$$

We have also

$$
\varphi(x)<\psi(x), \quad x_{1} \leqq x<x_{2},
$$

in virtue of (12) and of the fact that by (31) and (32) this inequality holds in some left neighborhood of $x_{2}$.

But from (14), (33) and (34) we have

$$
\psi\left(x_{0}\right) \leqq \phi\left(x_{0}\right), \quad \psi\left(x_{0}\right)>\phi\left(x_{0}\right)=\phi\left(x_{0}\right)
$$

and this is absurd. Therefore (31) is false and (29) must hold. By (11) we must have

$$
\varphi(x)=\psi(x) \leqq \phi(x), \quad x_{1} \leqq x \leqq x_{3} .
$$

Suppose now

$$
\varphi\left(x_{2}\right)<\phi\left(x_{2}\right) .
$$

Then it is easy to see, in virtue of (11), that there must be a point $x_{0}<\xi<x_{2}$ such that

$$
\varphi(\xi)=\psi(\xi) .
$$

Therefore we have, by (12),

$$
\varphi(x)<\psi(x) \leqq \phi(x), \quad \xi<x<x_{3} .
$$

Combining (28) and (37) we have

$$
\varphi(x) \leqq \phi(x), \quad x_{1} \leqq x \leqq x_{3} .
$$

Then the property $(\mathrm{P})$ holds on the closed interval $\left[x_{1}, x_{3}\right]$ and our 
lemma is demonstrated.

From this lemma follows immediately the proof of part (b) of our theorem. Indeed let $\alpha, \beta$ be two arbitrary numbers satisfying the condition $a<\alpha<\beta<b$. We must demonstrate that the $\theta(x) \in\{F(x)\}$ defined by

$$
\theta(\alpha)=\phi(\alpha), \quad \theta(\beta)=\phi(\beta)
$$

is such that

$$
\phi(x) \leqq \theta(x),
$$$$
\alpha \leqq x \leqq \beta
$$

In fact suppose there is an $\alpha<\lambda<\beta$ such that

$$
\phi(\lambda)>\theta(\lambda)
$$

and consider the function $\omega(x) \in\{F(x)\}$ such that

$$
\omega(\lambda)=\phi(\lambda), \quad \omega^{\prime}(\lambda)=\phi^{\prime}(\lambda) .
$$

By Lemma 2 we know that

$$
\omega(\alpha) \leqq \phi(\alpha)=\theta(\alpha), \quad \omega(\beta) \leqq \phi(\beta)=\theta(\beta) .
$$

From (40) and (41) we conclude the existence of two numbers $p, q$, $\alpha<p<\lambda<q<\beta$, such that

$$
\theta(p)=\omega(p), \quad \theta(q)=\omega(q)
$$

and this contradicts (12). Therefore (40) is false and (39) must hold. So condition (13) is sufficient to secure that $\phi(x)$ is a sub- $\{F(x)\}$ function. Hence Theorem 2 is proved.

REMARK. This theorem generalizes obviously the well known property of ordinary convex functions considered in the beginning. It also generalizes the result that the condition

$$
\phi^{\prime \prime}(x)+\phi(x) \geqq 0
$$

is necessary and sufficient in order that the twice differentiable function $\phi(x)$ be convex relative to the family of solutions of the equation

$$
y^{\prime \prime}+y=0
$$

( $x$ varying in an interval shorter than $\pi$ ) which is equivalent $[6$, p. 98 ; 7, p. 281] to a result due to G. Pólya.

5. A sharper result. A sharper result is expressed by the following theorem.

THEOREM 3. A sufficient condition in order that the twice differentiable 
function $\phi(x)$ be a strictly sub- $\{F(x)\}$ function is that

$$
\phi^{\prime \prime}>G\left[x, \phi, \phi^{\prime}\right], \quad a<x<b .
$$

Proof. The proof of this theorem is but slightly different from that of Theorem 2, part (b), and we shall only insist on the modifications. It follows easily from the following lemmas.

LEMma $1^{\prime}$. If $\left(13^{\prime}\right)$ holds, to every $a<x_{0}<b$ there exists a number $\nu>0$ such that

$$
\phi\left(x^{\prime}\right)-\bar{\varphi}\left(x^{\prime}\right)>0 \quad\left|x^{\prime}-x_{0}\right| \leqq \nu,\left|\bar{x}-x_{0}\right| \leqq \nu, x^{\prime} \neq \bar{x} .
$$

To demonstrate this lemma it is sufficient to observe that in virtue of $\left(13^{\prime}\right), \phi^{\prime \prime}\left(x_{0}\right)-\varphi^{\prime \prime}\left(x_{0}\right)>0$ so that by $(27)$ and from the arbitrariness of $\epsilon$ we have $\left(20^{\prime}\right)$.

Definition 2. Let us say that a closed interval has the property $\left(\mathrm{P}^{\prime}\right)$ when the graph of $\phi(x)$ lies above any integral curve of (9) which at a point of the arc of $\phi(x)$ corresponding to the interval has the same slope as $\phi(x)$-with the unique exception of the point of contact.

The above lemma states that each point $a<x_{0}<b$ is the center of a closed interval where the property $\left(\mathrm{P}^{\prime}\right)$ holds.

Lemma 2 . The property $\left(\mathrm{P}^{\prime}\right)$ holds on every closed interval $(\alpha, \beta)$, $a<\alpha \leqq x \leqq \beta<b$.

The demonstration is analogous to that of Lemma 2 and will not be given here.

The proof of Theorem 3 follows easily. Indeed let $\alpha, \beta$ be two arbitrary numbers satisfying the condition $a<\alpha<\beta<b$ and $\theta(x) \in\{F(x)\}$ defined by (38). We have shown that (39) holds. Now we shall show that we have

$$
\phi(x)<\theta(x), \quad \alpha<x<\beta .
$$

In fact, suppose there is $\alpha<\xi<\beta$ such that

$$
\phi(\xi)=\theta(\xi) \text {. }
$$

Then we have, by (39),

$$
\phi^{\prime}(\xi)=\theta^{\prime}(\xi) .
$$

By Lemma 2' we have that

$$
\theta(x)<\phi(x), \quad \alpha<x<\beta, x \neq \xi,
$$

and (46) contradicts (39). So (44) is false and (43) holds. The theorem is demonstrated.

REMARK. The condition $\left(13^{\prime}\right)$ is not necessary in order that $\phi(x)$ 
be a strictly sub- $\{F(x)\}$ function. This is not true even when the convexity is taken in the ordinary sense. For instance, $\phi(x)=x^{4}$, $-\infty<x<+\infty$, is obviously a strictly convex function but $\phi^{\prime \prime}(0)=0$.

6. The theorem of Tchaplygin. Consider the differential equation

$$
y^{\prime \prime}=p_{1} y^{\prime}+p_{2} y+q, \quad x \geqq x_{0},
$$

where $p_{1}(x), p_{2}(x)$ and $q(x)$ are continuous when $x \geqq x_{0}$ and let $y(x)$ be a solution of this equation such that

$$
y\left(x_{0}\right)=y_{0}, \quad y^{\prime}\left(x_{0}\right)=y_{0}^{\prime} .
$$

Suppose there exists a solution $u(x)$ of

$$
u^{\prime \prime}=p_{1} u^{\prime}+p_{2} u
$$

such that

$$
u(x) \neq 0,
$$$$
x_{0}<x<x_{1} .
$$

Let $u_{0}(x)$ be a solution of (49) such that $u_{0}\left(x_{0}\right)=0, u_{0}^{\prime}\left(x_{0}\right)=1$ and let $X\left(x_{0}\right)$ be the first zero of $u_{0}(x)$ to the right of $x_{0}$, if any such zero exists; otherwise let $X\left(x_{0}\right)=+\infty$. Then a recent theorem of $\mathrm{S}$. Tchaplygin, successively generalized by N. V. Petrov and J. E. Wilkins [3] states that:

(I) If $\phi(x)$ is such that

$$
\phi^{\prime \prime}>p_{1} \phi^{\prime}+p_{2} \phi+q, \quad x \geqq x_{0}, \phi\left(x_{0}\right)=y\left(x_{0}\right), \phi^{\prime}\left(x_{0}\right)=y^{\prime}\left(x_{0}\right)
$$

then

$$
\phi(x)>y(x), \quad x_{0}<x \leqq x_{1} .
$$

(II) The interval $x_{0}<x \leqq X\left(x_{0}\right)$ is the largest one in which the inequality (52) can be asserted to hold.

We shall prove that part (I) of this theorem can be deduced easily from Lemma $2^{\prime}$ and Lemma $1^{\prime}$.

In fact, from (50) it follows that in the strip $x_{0}<x<x_{1},-\infty<y$ $<+\infty$, the equation (47) has the property (12) that there is a unique solution passing through two arbitrary points with distinct $x$-coordinates. To see this known fact it is sufficient to observe that if $u(x)$ is a solution of (49) another independent solution of this equation is

$$
u(x) \int_{x_{0}}^{x} u^{-2}(t) \exp \left[\int_{t_{0}}^{t} p_{1}(t) d t\right] d t .
$$

Therefore, we have by Lemma $2^{\prime}$ that property $\left(\mathrm{P}^{\prime}\right)$ holds on every 
closed interval contained in $x_{0}<x<x_{1}$.

According to (51), there is a number $\xi<x_{1}$ such that

$$
\phi(x)>y(x), \quad x_{0}<x \leqq \xi .
$$

Let $g(x)$ be the solution of (47) such that $g(\xi)=\phi(\xi), g^{\prime}(\xi)=\phi^{\prime}(\xi)$. We have, by Lemma 2 ', that

$$
g(x)<\phi(x), \quad x_{0}<x<x_{1}, x \neq \xi .
$$

If it is true that

$$
g(x) \geqq y(x), \quad x_{0} \leqq x<\xi,
$$

we conclude by (53) and (54) that $g^{\prime}\left(x_{0}\right)=y^{\prime}\left(x_{0}\right)$, which is absurd. Then (55) is false and from the property (12) it follows that there exists a $\xi_{0}, x_{0}<\xi_{0}<\xi$, such that

$$
\begin{array}{cl}
g\left(\xi_{0}\right)=y\left(\xi_{0}\right), \quad g(x)<y(x) & \text { for } x_{0}<x<\xi_{0}, \\
g(x)>y(x) & \text { for } \xi_{0}<x<x_{1} .
\end{array}
$$

From (53), (54) and (56) it follows that

$$
y(x)<\phi(x),
$$$$
x_{0}<x<x_{1}
$$

So it is sufficient to demonstrate that

$$
y\left(x_{1}\right)<\phi\left(x_{1}\right)
$$

in order to state that (52) holds. But from (54) it follows immediately that

$$
g\left(x_{1}\right) \leqq \phi\left(x_{1}\right) .
$$

If $g\left(x_{1}\right)<\phi\left(x_{1}\right)$, then (58) follows from (56). Suppose now

$$
g\left(x_{1}\right)=\phi\left(x_{1}\right) .
$$

Using for the point $x_{1}$ the reasoning of Lemma $1^{\prime}$ we see that there is a point $\xi_{0}<\xi_{1}<x_{1}$ such that the solution $h(x)$ of (47) determined by $h\left(\xi_{1}\right)=\phi\left(\xi_{1}\right), h^{\prime}\left(\xi_{1}\right)=\phi^{\prime}\left(\xi_{1}\right)$ is such that

$$
h(x)<\phi(x), \quad \xi_{1}<x \leqq x_{1} .
$$

Then, by (54), (60) and (61), we have that there exists a point $\xi_{2}$, $\xi_{1}<\xi_{2}<x_{1}$, such that

$$
g\left(\xi_{2}\right)=h\left(\xi_{2}\right) .
$$

Repeating the reasoning which showed the falseness of (55) we conclude the existence of a number $\xi_{3}, \xi<\xi_{3}<\xi_{2}$, such that 


$$
g\left(\xi_{3}\right)=h\left(\xi_{3}\right) .
$$

But (62) and (63) are in contradiction with property (12). So (60) is false and (52) holds. Our statement is proved.

My thanks to Mrs. Marilia Chaves Peixoto.

\section{BIBLIOGRAPHY}

1. E. F. Beckenbach, Generalized convex functions, Bull. Amer. Math. Soc. vol. 43 (1937) pp. 363-371.

2. E. F. Beckenbach and R. H. Bing, On generalized convex functions, Trans. Amer. Math. Soc. vol. 58 (1945) pp. 220-230.

3. J. E. Wilkins, The converse of a theorem of Tchaplygin on differential inequalities, Bull. Amer. Math. Soc. vol. 53 (1947) pp. 126-129.

4. Mauricio Matos Peixoto, On the existence of derivative of generalized convex functions, Summa Brasiliensis Mathematicae vol. 2 fasc. 3 (to appear).

5. E. Picard, Traitê d'analyse, vol. 3, Paris, Gauthier-Villars, 1928. 1934.

6. G. H. Hardy, J. E. Littlewood, and G. P6lya, Inequalities, Cambridge, England,

7. G. Valiron, Fonctions convexes et fonctions entières, Bull. Soc. Math. France vol. 60 (1932) pp. 278-287.

\section{UNIVERSITY OF BRAZIL}

